\title{
Utility of the visual analog scale for the assessment of dental anxiety
}

\section{Przydatność skali wzrokowo-analogowej do oceny poziomu lęku stomatologicznego}

\author{
Monika U. Skowron ,A-D,F, Przemysław G. Nowak ${ }^{2, A, D, F}$, Włodzimierz Więckiewicz, ${ }^{1,-F}$, Martyna Waliczek², 2,E,F \\ 'Department of Dental Prosthetics, Wroclaw Medical University, Wroclaw, Poland \\ ${ }^{2}$ Department of Toxicology and Health Protection, Medical University of Silesia, Katowice, Poland \\ A - research concept and design; $\mathrm{B}$ - collection and/or assembly of data; $\mathrm{C}$ - data analysis and interpretation; \\ $\mathrm{D}$ - writing the article; $\mathrm{E}$ - critical revision of the article; $\mathrm{F}$ - final approval of the article
}

Address for correspondence

Monika Skowron

E-mail: nikaskylark@gmail.com

Funding sources

None declared

Conflict of interest

None declared

Received on July 25, 2017

Reviewed on September 12, 2017

Accepted on 0ctober 12, 2017

\begin{abstract}
Background. Psychometric scales can be used to assess the level of dental anxiety, but for practical reasons these are not routinely used in practice. This provides the opportunity to look for a simpler method that can be applied in the everyday work of a dentist.

Objectives. The study was designed to determine the utility of the visual analog scale (VAS) for dental anxiety assessment.

Material and methods. The study involved 315 patients who were awaiting their appointments; they were asked to complete a survey consisting of a scale and questionnaires: Modified Dental Rating Scale (MDAS), Kleinknecht's Dental Fear Survey (DFS), Dental Beliefs Survey (DBS), and Visual Analog Scale (VAS) of anxiety.

Results. An assessment of anxiety level measured by the MDAS scale showed that the majority of patients in the study group were patients with an average level of anxiety (68\%), $25 \%$ with low and $7 \%$ with high levels of anxiety (phobia). The DFS found that $45 \%$ were patients with low anxiety levels, $48 \%$ with an average level, and $7 \%$ with phobias. By using the DBS scale, it was observed that the vast majority of respondents showed average confidence (70\%), 26\% high trust and only 4\% did not trust the dentist. There is a high correlation between VAS scores and MDAS and DFS scales.
\end{abstract}

Conclusions. The VAS scale can be a simple and reliable tool for assessing anxiety in patients before dental surgery.

Key words: dental anxiety, dental treatment, visual analog scale

Słowa kluczowe: lęk dentystyczny, leczenie stomatologiczne, skala wzrokowo-analogowa

DOI

$10.17219 / \mathrm{dmp} / 78544$

Copyright

○ 2017 by Wroclaw Medical University

and Polish Dental Society

This is an article distributed under the terms of the

Creative Commons Attribution Non-Commercial License

(http://creativecommons.org/licenses/by-nc-nd/4.0/) 
Dentophobia is manifested by very intense anxiety, accompanied by strong symptoms from the autonomic nervous system. The consequence of increased anxiety is a worsening of oral hygiene, because a patient who is "obsessively" afraid of dental treatment tends to avoid appointments, and if the patient decides to be treated, it is a very traumatic experience for the him or her, and this adversely affects and aggravates their general well-being and quality of life. ${ }^{1}$ Irregular visits or discontinuations undermine the effectiveness of therapy and rehabilitation and can delay recovery. ${ }^{2}$ Given the above, an assessment of dental anxiety level provides very valuable information for the dentist, because it can affect the entire process of treatment and patient care, including the appointment schedule, an adaptation visit, plan of and proposed treatments, anesthesia techniques or the necessity of pharmacological premedication. Among the many methods most commonly used are psychometric scales, which are standard patient-completed questionnaires. We can mention here the Corah Dental Anxiety Scale (CDAS), Modified Dental Anxiety Scale (MDAS), Kleinknecht's Dental Fear Survey (DFS), and others. ${ }^{3-6}$ Despite the availability of these tools for evaluating anxiety, practice indicates that they are rarely used in clinical settings because filling out questionnaires by patients as well as their interpretation by physicians is time-consuming. The above factors highlight the lack of a simple and "friendly" but credible tool that can be used in the daily work of the dentist to assess the severity of anxiety.

Numerous studies indicate that the primary source of dental anxiety is fear of pain during extraction, anesthesia or drilling. ${ }^{7}$ Simplified scales are used to assess the severity of pain in many medical fields, but primarily in oncology, palliative medicine and anesthesiology. These allow the patient to quickly and reliably record the intensity of the pain. These scales include the Visual Analog Scale (VAS). The VAS is a segment of $10 \mathrm{~cm}$ : from 0 (no pain) to 10 (unbearable pain). The patient's task is to mark with a vertical line the point on the scale corresponding to the current intensity of pain. Similar to the subjective feeling of pain is the feeling of fear. Unfortunately, only individual reports can be found in the available literature in which the VAS (taken from the pain assessment methodology) has been used to assess anxiety in hospitalized patients awaiting surgery. ${ }^{8}$ In the Polish literature, however, there is no study on the use of VAS to evaluate dental anxiety.

\section{Objectives}

The purpose of the study was to: (1) estimate the correlation between the VAS and typical MDAS and DFS dental scales; and (2) determine the suitability of the VAS for evaluating anxiety levels in adult patients prior to dental treatment.

\section{Materials and methods}

The study included 315 patients aged 18-81 who reported to the dental office at Non-Public Health Care Facility at Dworcowa 1 Str. 46-020 Czarnowąsy. The questionnaire was delivered daily to the first 2 to 3 patients reporting for dental treatment. The study was conducted between January and December 2015. Respondents filled out the questionnaires themselves. Mid-level medical staff distributed the questionnaires, and their participation in the study was limited to the minimum, i.e. explaining the aims and purpose of the survey, and being told how to complete and distribute questionnaires.

Patients awaiting a dentist's appointment were asked to complete a survey containing (1) demographics, (2) questions related to the source of the most unpleasant experiences during dental treatment, (3) questions associated with the reason for the dental appointment, (4) a modified scale of dental anxiety assessment (MDAS), (5) a scale of dental fear evaluation (DFS), (6) a scale of trust in the dentist (Dental Beliefs Scale, DBS), and (7) to mark the level of anxiety on the VAS scale.

The MDAS consists of 5 questions that relate to the specific circumstances related to the dental treatment, about which a choice is made, categorized on the Likert scale of 5 , to assess anxiety in a given situation. For a specific statement, the patient was given the choice of one of the following answers: relaxed (1 point), a little restless ( 2 points), tense (3 points), nervous ( 4 points), and very upset, sweating, physically sick (5 points). The results were in the range of 5-25 points, and the higher the result, the higher the anxiety. This scale was originally dichotomous, i.e. the results of 19 points or more were defined as very high anxiety or phobia, while results below this value indicated either the presence of anxiety or the lack thereof. At present, there are also studies in which the raw data is categorized at 3 or 4 levels, and these were also used in this study to generalize (in graphical form) the intensification of the dental anxiety of the studied population. There were 4 categories: no anxiety ( 5 points), low anxiety (6-10 points), moderate anxiety (11-18 points), and high anxiety (19-25 points). In the literature, there is a great deal of freedom in terms of the point ranges that define a given category. ${ }^{6,9-11}$ MDAS has been translated into the Polish language and validated by Dubielecka et al. ${ }^{12}$

DFS is used to assess the level of dental fear in 20 typical situations, such as appointments, listening to the sound of the drill or seeing the needle for anesthesia - rated on the Likert scale from 1 (no fear) to 5 (very anxious). The scores range from 20 to 100 . The scale was also originally dichotomized, i.e. results of 63 points or higher refer to patients with very high anxiety (phobia), while results below 63 points indicate the presence or absence of anxiety. Also, as with the MDAS scale, one can find studies in which the raw results are categorized at 3 or 4 levels, and these were also used in this study. The following was used: 
no fear (20 points), low anxiety (21-41 points), moderate anxiety (42-62 points), and high anxiety (63-100 points). In the literature, as in the case of the MDAS scale, there is a great deal of freedom in terms of the point ranges for a given category. ${ }^{4,5,12}$ DFS has not been validated in the Polish language, though there is a bulk of studies that confirm its utility in dental fear assessment. ${ }^{13,14}$

It must be added that terms like dental fear and dental anxiety are frequently used indiscriminately, but they represent different progressive degrees of the same psychological condition. Dental fear refers to a normal unpleasant emotional reaction to specific threatening stimuli occurring in situations during dental treatment. Conversely, dental anxiety is an excessive and unreasonable negative emotional state experienced by dental patients and results from the premonition that something undesirable is about to happen. Altogether, the results from the DFS and MDAS are qualitatively slightly different but, as mentioned above, used in dentistry interchangeably.

The DBS scale of trust in a dentist developed by the Getz team is comprised of 15 questions on issues such as information provided by the doctor, behavior of the doctor, qualifications, competencies, and the patient's ability to ask questions. Some researchers use a scale of 16 questions. ${ }^{15,16}$ In our study, a 15 item Getz scale was employed. Patients assess their trust in the dentist using a 5-point Likert scale, where the patient can agree with a specific question fully ( 5 points), mostly ( 4 points), partly ( 3 points), to a lesser extent ( 2 points) or disagree (1 point). The sum of the points, in the range of 15-75, gives an overview of the patient's subjective perceptions about the dentist and the course of the appointment. The raw results have allowed 3 categories to be identified: high confidence level (15-34 points), medium confidence level (35-54 points), and low confidence level (55-75 points). In the further statistical analysis, as with other scales (MDAS, DFS), the raw results were used.

Respondents were also asked to highlight the severity of anxiety on the VAS scale at that moment, i.e. immediately prior to the visit to the dental office. The VAS scale is a $100-\mathrm{mm}$ segment where the patient's vertical line indicates the level of his or her anxiety. The results were assessed by measuring (in $\mathrm{mm}$ ) the distance from the beginning of the scale to the spot marked by the patient. The value of "0" means no fear, while " 100 " is the maximum intensity of anxiety that a person can imagine.

\section{Statistical analysis}

Statistical analysis was performed with STATISTICA v. 8.0 PL (StatSoft, Inc., Tulsa, USA) and MS Excel 2007. The analysis used parametric tests, i.e. Student's t-test, one-way analysis of variance and the Newman-Keulus post-hoc test. Non-parametric data was analyzed with the $\chi^{2}$ test. Spearman's test was used to evaluate the cor- relation. The $\mathrm{p}<0.05$ or 0.001 values were statistically significant. Interpretation of the correlation coefficient was based on the following classification: $r=0-$ no correlation; $0.0<\mathrm{r} \leq 0.2$ - very weak correlation; $0.2<\mathrm{r} \leq 0.4$ - weak correlation; $0.4<\mathrm{r} \leq 0.7$ - average correlation; $0.7<\mathrm{r} \leq 0.9$ - high correlation; $0.9<\mathrm{r} \leq 1.0$ - very high correlation; $r=1$ - full correlation.

\section{Results}

A total of 315 people participated in the survey of anxiety intensity in patients before visiting the dental office; 9 questionnaires were completed incorrectly; therefore, 306 surveys were included in the further analysis. Of the surveyed patients, women accounted for $52 \%$ and men $48 \%$. Regarding age categories, the largest groups of respondents were under 30 years (26\%) and $41-50$ years (24\%). Patients aged 31-40 years accounted for $18 \%$ of the respondents, $51-60$ years $14 \%$ and over 60 years $17 \%$. Of the respondents, 33\% had higher education, $34 \%$ vocational education, 29\% secondary education and 4\% primary education only.

By analyzing the reasons for the respondents' appointments with the dentist, $48 \%$ reported tooth treatment, $14 \%$ tooth extraction, $10 \%$ hygienic treatment, $4 \%$ surgery, $11 \%$ a general check-up, and $12 \%$ could not clearly determine the reason for the appointment or the type of treatment. As the source of the most unpleasant experiences during the appointment, $77 \%$ of the respondents described pain during the procedure, $12 \%$ waiting for the procedure, $5 \%$ pain during anesthesia, $3 \%$ reported an inappropriate approach by the physician, and $3 \%$ other reasons not mentioned in the survey. The majority of respondents had average confidence (70\%), 26\% had high trust, and only $4 \%$ did not trust the dentist.

The level of anxiety (categorized) measured by the MDAS scale showed that the majority of patients in the respondent group were people with moderate anxiety levels (68\%), 25\% with low and 7\% with high levels of anxiety (phobia) (Fig. 1). The assessment of anxiety level measured by the DFS scale showed that in the study group, respondents with low anxiety levels accounted for $45 \%$, with average anxiety $48 \%$, and those with high anxiety

@ high level of anxiety (phobia) $\quad$ average level of anxiety $\quad$ low level of anxiety

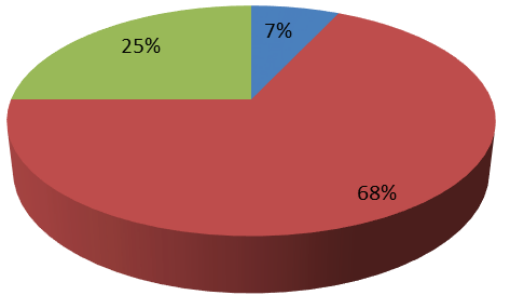

Fig. 1. The level of anxiety (categorized) measured by the MDAS 
- high level of anxiety (phobia) = average level of anxiety a low level of anxiety

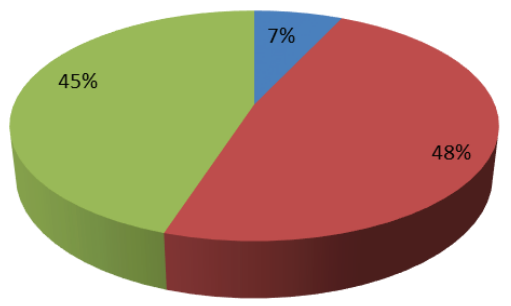

Fig. 2. The level of anxiety (categorized) measured by the DFS

(phobia) 7\% (Fig. 2). Using the scales to assess MDAS and VAS anxiety, it was found that sex remained unaffected by the test parameter This is similar to the parameter of trusting a dentist as rated by the DBS scale. The intensity of anxiety was significantly higher in women based on the results of the DFS scale (Table 1). At the same time, it was shown that with increasing age, anxiety levels rose, but this dependency only concerned the comparison of people over the age of 60 with respondents under 30 years of age (MDAS and DFS questionnaires) or $51-60$ vs $31-40$ (VAS scale).

Age did not influence the aspect of trust in the dentist (Table 2). People with higher education showed significantly lower anxiety levels than those with primary, vocational or secondary education (MDAS, DFS and VAS scales). The type of education did not affect the confidence in the dentist measured by the DBS questionnaire. The type of procedure affected the severity of anxiety, with those seeking tooth extraction or those who could not give a reason for an appointment exhibiting a higher anxiety level compared to patients attending a general check-up, dental treatment, or hygienic treatment.

Table 1. Analysis of variables with regard to gender

\begin{tabular}{|l|c|c|c|c|c|}
\hline \multirow{2}{*}{ Variables } & $\begin{array}{c}\text { Female } \\
(\mathrm{n}=158)\end{array}$ & $\begin{array}{c}\text { Male } \\
(\mathrm{n}=148)\end{array}$ & \multicolumn{3}{|c|}{ Analysis } \\
\cline { 2 - 7 } & average & average & factor & p-value & type of test \\
mDAS & $13.13 \pm 3.70$ & $12.62 \pm 3.07$ & $\mathrm{t}=1.309$ & 0.191 & \\
DFS & $45.61 \pm 13.82^{*}$ & $42.24 \pm 10.45$ & $\mathrm{t}=2.393$ & $<0.05$ & test \\
VAS & $34.37 \pm 19.80$ & $31.77 \pm 19.81$ & $\mathrm{t}=1.146$ & 0.253 & t-Studenta \\
DBS & $39.41 \pm 8.92$ & $38.85 \pm 9.62$ & $\mathrm{t}=0.535$ & 0.593 & \\
\hline
\end{tabular}

${ }^{*} p<0.05$
There was a strong correlation between VAS scores and MDAS scores for dental anxiety (Spearman's $r=0.7616$; $\mathrm{p}<0.001)$. There was a strong correlation between VAS scores and DFS questionnaire scores for dental anxiety (Spearman's $\mathrm{r}=0.7674 ; \mathrm{p}<0.001$ ). There was a weak correlation between trust in the dentist assessed on the DBS scale and anxiety levels determined on the VAS scale (Spearman's $\mathrm{r}=0.2780$; $\mathrm{p}<0.001$ ). There was a strong correlation between MDAS and DFS dental anxiety scores (Spearman's $r=0.7686$; $\mathrm{p}<0.001$ ). There was a weak correlation between the results of the dental anxiety MDAS scores and the trust in the dentist scores assessed on the DBS scale (Spearman's $r=0.2743$; $p<0.001$ ). There was an average correlation between DFS dental anxiety scores and trust in the dentist scores assessed on the DBS scale (Spearman's $\mathrm{r}=0.11617$; $\mathrm{p}<0.001)$.

\section{Discussion}

Dental anxiety can, to a large extent, be "modeled" by environmental factors and prior experience. Significant factors are early and negative medical experiences, family impact, age and cause of the first visit to the dentist. Studies show that fear of dental treatment in adults can be acquired and learned in childhood. Considering the question of dental anxiety, we should consider the patient's trust in the doctor and the whole dental treatment process a trust built from early childhood. This is confirmed by, among others, the Olszewska study, which showed such a relationship; namely, patients declaring a high level of anxiety were characterized by low confidence in the dentist. ${ }^{17}$ Results confirmed that the most important positive qualities of a physician for patients are competence and avoidance of pain, while the worst is awkwardness, roughness and lack of respect and haste.

Wilk-Sieczak and Gmyrek-Marciniak assessed the effect of family attitudes on children's behavior during their first visit to the dentist, declaring that the majority of surveyed mothers and caregivers declared high $(61.4 \%$ and $58.6 \%$, respectively) and average (27.2\% and 35.8\%) trust in the dentist. ${ }^{15}$ At the same time, it was shown that a low level of trust in the dentist on the part of the mothers was significantly correlated with difficult behavior during the first visit to the dentist. The results of this study indicate

Table 2. Analysis of variables with regard to age

\begin{tabular}{|c|c|c|c|c|c|c|c|c|}
\hline \multirow{3}{*}{ Variables } & \multicolumn{8}{|c|}{ Age in year divided into groups } \\
\hline & group 1 & group 2 & group 3 & group 4 & group 5 & & analysis & \\
\hline & $<30(n=81)$ & $31-40(n=56)$ & $41-50(n=73)$ & $51-60(n=43)$ & $>60(n=53)$ & factor & $p$-value & type of test \\
\hline MDAS & $12.21 \pm 3.72$ & $12.36 \pm 3.10$ & $12.92 \pm 3.47$ & $13.56 \pm 2.94$ & $13.89 \pm 3.30^{*}$ & $F=2.909$ & $<0.05$ & \multirow{4}{*}{ ANOVA } \\
\hline DFS & $41.00 \pm 13.29$ & $42.34 \pm 11.95$ & $44.99 \pm 13.04$ & $45.61 \pm 10.09$ & $47.59 \pm 11.32^{*}$ & $F=3.745$ & $<0.05$ & \\
\hline VAS & $30.09 \pm 21.02$ & $27.61 \pm 16.64$ & $36.11 \pm 21.16$ & $35.61 \pm 17.67^{*}$ & $37.38 \pm 19.39$ & $F=2.815$ & $<0.05$ & \\
\hline DBS & $37.21 \pm 10.26$ & $38.91 \pm 7.39$ & $39.56 \pm 8.73$ & $39.21 \pm 9.59$ & $41.72 \pm 9.47$ & $F=1.982$ & 0.097 & \\
\hline
\end{tabular}

Groups:1/5; $2 / 4 * p<0.05$ 
that sex, age, education and type of procedure remained virtually unaffected by the degree of patient confidence in the physician. However, there is a positive correlation between the results of the MDAS, DFS and VAS scores and the trust in the physician. Patients who trust a doctor show less aggravation and vice versa, while a distrustful person is characterized by a higher intensity of anxiety. Similar correlations have been observed by other authors, e.g. Kowalińska-Kania et al. confirmed (in children aged 10-15 years) a moderate correlation between anxiety levels measured on the NRS, CDAS and CFSS-DS numerical scales and the degree of confidence measured by the DBS questionnaire. ${ }^{18}$ The cited authors also noted that with increased confidence in the dentist, the anxiety of the examined children decreases, which is also consistent with the results of other researchers studying similar relationships in a Lithuanian child population. ${ }^{19}$

There are many papers analyzing the impact of variables such as age, sex, education, socioeconomic status, or type of procedure on dental anxiety. ${ }^{20}$ Olszewska et al. found that women exhibited a higher degree of anxiety than men, similar to those with primary education compared to patients with higher education. ${ }^{21}$ The greatest anxiety was aroused in patients by such procedures as grinding teeth under the crown or prosthetic bridge and root canal treatment (both also considered the most painful), and the least by removal of tartar. Similar results were obtained by Firat et al. in a Turkish population. ${ }^{22}$ On the MDAS scale, women in this study scored significantly higher than men, while DFS scores were negatively correlated with education (lower education - higher anxiety). ${ }^{22}$ These results are consistent with our findings.

When analyzing the problem of dental anxiety, the most common source of anxiety in dentistry is fear of pain during the procedure. ${ }^{23}$ In our study, the source of the most unpleasant experiences during a visit to a dental clinic was pain during the procedure (77\%), waiting for surgery (12\%), pain during anesthesia (5\%), the wrong approach by the doctor $(3 \%)$ and other reasons not mentioned in the survey (3\%). Interestingly, further analysis has shown that the type of procedure also affects anxiety. So, those attending for tooth extraction, or those who could not give a reason for a visit exhibited a higher anxiety level compared to patients who had come for a general checkup or hygienic treatment.

Our results are partially consistent with the results of other authors, for example Walawender et al., who found that pain during surgery was the source of the most unpleasant sensations for $41 \%$ of respondents, anesthesia pain for $22 \%$, incorrect treatment for $17 \%$, prolonged waiting for surgery for $13 \%$, and $7 \%$ indicated other causes. Borowy et al., when analyzing the intensity of dental anxiety and attitudes toward the dentist (among university students in Kraków), also showed that the most unpleasant experience during the visit was pain during the procedure (48.9\%) or a long wait for the visit $(21.7 \%) .{ }^{13}$ The remain- ing responses pointed to pain during anesthesia, incorrect approach by the doctor, and reasons described as "other".

In conclusion, in most studies, patients point to pain during the procedure as a source of unpleasant sensations during a dentist visit. In our study, using both MDAS and DFS scales, dentophobia was reported by about $7 \%$ of patients. Similar results $(7.3 \%)$ were obtained by Nicolas et al. ${ }^{24}$ (France), while the least frequent incidence of dentophobia occurred in Denmark, i.e. $4 \% .{ }^{25}$ Women and the elderly show a higher level of anxiety, which was also confirmed in our study. (Tables 1 and 2). Dentophobia was reported in $9.49 \%$ of women and only $4.05 \%$ of men (MDAS questionnaire, $\left.\chi^{2}=3.537 ; \mathrm{p}=0.059\right)$ or $11.39 \%$ of women and only $2.03 \%$ of men (DFS questionnaire $\chi^{2}=10.486$; $\mathrm{p}<0.05$ ) (results not graphically presented). The above observations coincide with the reports of other authors. ${ }^{11}$

Considering the importance of anxiety in dentistry, it is important to identify patients with very high (phobia) and high levels of dental anxiety, as this can have a positive effect on the final result. In our study, the VAS scale was derived from the pain assessment methodology, and 2 additional scales, MDAS and DFS, were used for dental patients only. The raw results of the MDAS and DFS questionnaires were categorized, which allowed the population to be divided into 3 groups, i.e. individuals with low, medium and high levels of anxiety. It was found that according to the MDAS scale, the majority of patients $(68 \%)$ in the respondent group were characterized by average anxiety levels, $25 \%$ with low and $7 \%$ with high levels of anxiety (phobia). The anxiety level measured, in turn, with the DFS scale showed that $45 \%$ of the respondents were characterized by low anxiety levels, $48 \%$ with average, and $7 \%$ with high-anxiety (phobia).

Kaczmarek et al. assessed the aggravation of anxiety among students of medicine and dentistry (using the MDAS scale) and confirmed that, in the respondent group, the highest number of people exhibited an average level of anxiety, i.e. $60.6 \%$ (medical students), and $47.2 \%$ (dental students), while for the low anxiety level it was 18.2\% (medical students) and 43.1\% (dental students) and high levels of anxiety (phobia) were $21.2 \%$ and $9.7 \%$, respectively. ${ }^{9}$ The same authors also used the second scale of dental anxiety assessment, i.e. DAS, which is a modification of MDAS. In spite of the similarity between these scales, they obtained a slightly different profile for the severity of anxiety, but, as they state, the differences were not statistically significant. The above results are mostly consistent with the results of our study, i.e. the prevalence of patients with moderate anxiety. In our study, the distribution of MDAS and DFS respondents to the respective anxiety groups was different, but in this case the differences were statistically significant.

Since the DFS questionnaire contains many more questions than the MDAS questionnaire, it is characterized by a higher sensitivity,so despite the high correlation, these research tools slightly differentially graduated the severity 
of dental disease in patients. Further studies have shown that there is a high correlation between VAS scores and the scales, which, as previously mentioned, are only used for dental anxiety assessment: MDAS and DFS. ${ }^{26}$

In the available literature, there is virtually no study that has addressed this problem. Only a handful of studies can be found in which the VAS scale (a "tool" taken from the methodology of assessing the severity of pain) has been used to measure anxiety in patients prior to a procedure. Thus, Millar et al. assessed the utility of VAS for anxiety assessment in a group of 44 preoperative patients, comparing the results obtained with the State-Trait Anxiety Inventory (STAI) and the results of the Hospital Anxiety and Depression Scale. ${ }^{27}$ The obtained relationships have shown that the VAS scale can be regarded as a reliable "tool" for assessing anxiety, but there are some limitations, i.e. the patient's uncertainty as to the correct answer or the tendency to avoid stress evaluations is revealed. The authors termed this "the impact of the central tendency" on the results. Similar issues were also studied by Kindler et al., who in a much larger group of patients (734), immediately prior to a procedure and anesthesia, assessed anxiety with the use of the VAS scale and the STAI questionnaire. ${ }^{28}$ These researchers also demonstrated a positive correlation between VAS and STAI scores.

Also, in the Polish literature you can find one publication from this range: Romanik et al. assessed the severity of anxiety in 38 patients awaiting a procedure using the STAI Inventory and the VAS scale. ${ }^{29}$ They stated that those who declared that they were afraid of surgery indicated a length of $3.7 \pm 2.6 \mathrm{~mm}$ on the VAS scale, while those who were not afraid $-1.5 \pm 1.7 \mathrm{~mm}$. The VAS scale has been used to evaluate anxiety in patients in a dental practice in only one publication, Bahammam and Hassan, by validating MDAS scale in the Arabic language. They recruited 486 patients, and after analysis they found that the Arabic version of MDAS is characterized by high reliability and accuracy. ${ }^{30}$ By plotting the Receiver Operating Characteristic curve (ROC), the cutoff point for the presence of very high dental anxiety (phobia) is 16 points or more. On the other hand, the assessment of anxiety on the VAS scale showed that the recruited patients were characterized by an average anxiety level $(55.74 \pm 17.46 \mathrm{~mm})$ and the results corresponded very well to MDAS scores.

Meisel-Denes et al. used the VAS scale, which is in some sense analogous to the numerical scale (NRS), in their study to assess pain and anxiety in sedation with nitrous oxide. ${ }^{31}$ Walawender et al. also evaluated dental anxiety in adult patients seeking dental surgery using the NRS scale and the results were compared to the STAI Inventory (general anxiety assessment) and CDAS questionnaire. ${ }^{24}$ These authors showed a high correlation between NRS and CDAS and a moderate correlation with the L-state STAI scale. Similar findings have been made by other researchers using NRS and CDAS and CFSS-DS scales, assessing anxiety in children aged $10-15$ years. ${ }^{17}$
In summary, clinical experience so far has shown that the currently-available scales for dental anxiety evaluation are rarely used under typical dental practice conditions. The results show that the VAS scale provides the opportunity for a quick and reliable assessment of the degree of anxiety in adult patients registered with a dental practice, and the unmatched benefits of this method are the short test time and simple interpretation of results.

\section{Conclusions}

Age, sex, education, and type of procedure have an impact on the severity of anxiety concerning dental treatment. A high degree of correlation was found between the results of the MDAS scale and DFS dental anxiety scores and VAS scale results. The VAS scale can be a simple and yet reliable tool for assessing anxiety in patients before dental surgery.

\section{References}

1. Kisely S. No mental health without oral health. Can J Psych. 2016;61:277-282

2. Boman UW, Wennström A, Stenman U, Hakeberg M. Oral healthrelated quality of life, sense of coherence and dental anxiety: An epidemiological cross-sectional study of middle-aged women. BMC Oral Health. 2012;12:14.

3. Corah N.L. Development of a dental anxiety scale. J Dent Res. 1969;48:596.

4. Kleinknecht RA, Klepac RK, Alexander LD. Origins and characteristics of fear of dentistry. J Am Dent Assoc. 1973;86:842-848.

5. Kleinknecht RA, Thorndike RM, McGlynn FD, Harkavy J. Factor analysis of the dental fear survey with cross-validation. J Am Dent Assoc. 1984;108:59-61.

6. Humphris GM, Morrison T, Lindsay SJ. The modified dental anxiety scale: Validation and United Kingdom norms. Commun Dent Health. 1995;12:143-150.

7. Walawender I, Roczniak W, Nowak D, et al. Applicability of the numeric scale for anxiety evaluation in patients undergoing dental treatment. Dent Med Prob. 2015;52:205-214.

8. Hjermstad MJ, Fayers PM, Haugen DF, et al. Studies comparing Numerical Rating Scales, Verbal Rating Scales, and Visual Analogue Scales for assessment of pain intensity in adults: A systematic literature review. J Pain Symptom Manage. 2011;41:1073-1093.

9. Kaczmarek U, Mysiak-Dębska M, Dębska K, Grzebieluch W. Dental anxiety in students of the first years of the study of dentistry and medicine faculties. Dent Med Probl. 2010;47:343-349 [in Polish].

10. Hierons RJ, Dorman ML, Wilson K, Averley P, Girdler N. Investigation of inhalational conscious sedation as a tool for reducing anxiety in adults undergoing exodontia. Br Dent J. 2012;213:15-19.

11. Saatchi M, Abtahi M, Mohammadi G, Mirdamadi M, Binandeh ES. The prevalence of dental anxiety and fear in patients referred to Isfahan Dental School, Iran. Dent Res J (Isfahan). 2015:12:248-253.

12. Dubielecka M, Rusyan E, Panczyk M, Mielczarek A. A preliminary assessment of the usefulness of the Polish language version of MDAS scale for the estimation of dental treatment anxiety levels among adults in Warsaw. Nowa Stomatol. 2016;21:4,203-207 [in Polish].

13. Borowy M, Kowalewska K, Mazur A. Assessment of the level of anxiety and attitude toward to the dentist among the students of Cracow universities. Poradnik Stomatol. 2011;11:230-234 [in Polish].

14. Merks K, Jaworska-Zaremba M. Fábián TK, Mierzwińska-Nastalska E. The analysis of dental fear scores in the Polish minority living in Hungary. Prot Stomatol. 2010;57:102-111 [in Polish].

15. Wilk-Sieczak B, Gmyrek-Marciniak A. Children's behaviour during their dental adaptation visits and their families' attitudes towards dental treatment. Dent Med Probl. 2005;42:573-580 [in Polish]. 
16. Wilk-Sieczak B, Zakrzewski M, Chmielewska-Łuczak D. Mothers fear of dental treatment and the cause of the first visits of the child, and factors predicting negative preschool child's attitudes during dental treatment. Dent Med Probl. 2005;42:77-82 [in Polish].

17. Olszewska I. Anxiety and belief in dentist-patient relations. Magazyn Stomatol. 2001;11:1,54-59 [in Polish].

18. Kowalińska-Kania M, Nowak D, et al. Numerical scale for anxiety assessment in 10-15 years children undergoing dental treatment. Dent Med Probl. 2015;52:309-315.

19. Recine R. Prevalence of dental fear among Vilnus pupils aged 12 to 15 years. Stomatolog Baltic Dent Maxillofac. 2003;5:52-56.

20. Armfield JM, Spencer AJ, Stewart JF. Dental fear in Australia: Who's afraid of the dentist? Aust Dent J. 2006;51:78-85.

21. Olszewska I, Żarow M, Gofroń B, Paczyńska P. Anxiety level analysis before dental treatment. Magazyn Stomatol. 2000;10:7-8,58-62 [in Polish].

22. Firat D, Tunc EP, Sar V. Dental anxiety among adults in Turkey. J Contemp Dent Pract. 2006;7:75-82.

23. Appukuttan DP, Tadepalli A, Cholan PK, Subramanian S, Vinayagavel M. Prevalence of dental anxiety among patients attending a dental educational institution in Chennai, India questionnaire based study. Oral Health Dent Manag. 2013;12:289-294.

24. Nicolas E, Collado V, Faulks D, Bullier B, Hennequin MA. National cross-sectional survey of dental anxiety in the French adult population. BMC Oral Health, 2007;7:12.

25. Moore R, Birn H, Kirkegaard E, Brødsgaard I, Scheutz F. Prevalence and characteristics of dental anxiety in Danish adults. Community Dent Oral Epidemiol. 1993;21:292-296.

26. Tunc EP, Firat D, Onur OD, Sar V. Reliability and validity of the Modified Dental Anxiety Scale (MDAS) in a Turkish population. Community Dent Oral Epidemiol. 2005;33:357-362.

27. Millar K, Jelicic M, Bonke B, Asbury AJ. Assessment of preoperative anxiety: Comparison of measures in patients awaiting surgery for breast cancer. Br J Anaesth. 1995;74:180-183.

28. Kindler $\mathrm{CH}$, Harms C, Amsler F, Ihde-Scholl T, Scheidegger D. The visual analog scale allows effective measurement of preoperative anxiety and detection of patients' anesthetic concerns. Anesth Analg. 2000;90:706-712.

29. Romanik W, Kański A, Soluch P, Szymańska O. Questionnaires and declarative level of anxiety of patients before surgery. Anest Int Ter. 2009;2:94-99 [in Polish].

30. Bahammam MA, Hassan MH. Validity and reliability of an Arabic version of the modified dental anxiety scale in Saudi adults. Saudi Med J. 2014;35:1384-1389.

31. Meisel-Denes J, Pregiel B, Sulka A. Use of the nitric oxide for dental treatment. TPS, 2014;6:95-100 [in Polish]. 
